\title{
Apoptosis is a major cause of so-called "caseous necrosis" in mycobacterial granulomas in HIV- infected patients
}

\author{
A S-Y Leong, ${ }^{1}$ P Wannakrairot, ${ }^{2}$ T Y-M Leong ${ }^{3}$
}

\begin{abstract}
${ }^{1}$ Division of Anatomical Pathology, Hunter Area Pathology Service and University of Newcastle, Australia 2310;

${ }^{2}$ Department of Pathology, Faculty of Medicine, Chulalongkorn University, Bangkok, Thailand; ${ }^{3}$ Victorian Cytology Service, Melbourne, Australia 3001
\end{abstract}

Correspondence to: Professor Anthony S-Y Leong, Hunter Area Pathology Service, Locked Bag 1, HRMC Newcastle, Australia 2310; aleong@mail.newcastle.edu.au

Accepted 15 August 2007 Published Online First 30 August 2007

\begin{abstract}
Aim: To demonstrate that so-called "caseous necrosis" is the result of apoptosis and investigate the association of $B$ and $T$ cells, and macrophages with the granulomas and their relationship to some apoptosis-related proteins.

Methods: Cervical lymph node biopsy specimens from 55 HIV-infected Thai patients with caseating granulomas, confluent caseating granulomas, sarcoid-like granulomas, foamy macrophage response, pseudo-inflammatory tumour response or non-specific lymphoid hyperplasia were examined histologically and for apoptosis by immunostaining for caspase 3 and TUNEL. Classic tuberculoid caseating granulomas in cervical lymph node and lungs from non-HIV-infected patients were also stained with caspase 3 .
\end{abstract}

Results: All areas of caseous necrosis frequently displayed extensive apoptosis that readily accounted for the so-called "necrosis". Small foci of apoptosis were present in the other reaction patterns and fibrotic granulomas often showed residual apoptosis. The extent of apoptosis was inversely related to the numbers of identifiable acid-fast bacilli; all epithelioid macrophages revealed strong immunoexpression of the pro-apoptotic proteins Bax and Fas, whereas the anti-apoptotic protein $\mathrm{Bcl}-2$ was not present. Apoptosis occurred in CD68+ macrophages and CD3+ CD8+ T cells; all nodes were deficient of $C D 4+$ cells. $C D 8+T$ cells were intimately related to the apoptotic foci, suggesting a role in the process, particularly in the absence of CD4+ cells. In nonHIV-infected cases, similar extensive apoptosis was confirmed with caspase 3 .

Conclusions: So-called "caseous necrosis" is shown for the first time to be the result of apoptosis. In the absence of CD4+ cells the findings negate many of the postulated mechanisms of apoptosis in the murine model and have implications for the treatment of mycobacterial infections.

A granulomatous response is the hallmark of mycobacterial infection. This inflammatory process is characterised by the presence of epithelioid macrophages that may be aggregated into localised collections of epithelioid cells or granulomas, or as diffuse infiltrates. Granulomas characteristically contain occasional macrophage polykaryons or multinucleated giant cells (Langhans' giant cells) and are surrounded by a cuff of variable numbers of small lymphocytes and occasional plasma cells with a peripheral rim of fibrosis. Mycobacterial granulomas may show central necrosis, so-called caseous necrosis because of its gross resemblance to lumpy cheese. Histologically, caseous necrosis has been described as a distinctive form of coagulative necrosis seemingly composed of amorphous eosinophilic granular debris of fragmented coagulated cells. ${ }^{1}$

Mycobacterium tuberculosis is the best-studied species of mycobacteria and is known to enter macrophages by endocytosis following the binding of opsonised mycobacteria mediated through monocyte mannose receptors and mycobacterial surface lipoarabinomannan. The bacteria have the ability to block fusion of phagosome with lysosome, thus preventing their destruction by lysosomal acid hydrolases. It has been speculated that this is the mechanism by which mycobacteria survive inside macrophages. T cells are essential for the recruitment and stimulation of macrophages to form granulomas. In particular, $\mathrm{T}_{\mathrm{H}} 1$ cells secrete interferon- $\gamma($ IFN- $\gamma)$ and other cytokines that convert macrophages to epithelioid histiocytes, which are thought to be necessary for the containment of the bacteria. The macrophages thus activated produce tumour necrosis factor- $\alpha$ (TNF- $\alpha$ ), which, in turn, recruits further monocytes to the site to form epithelioid histiocytes. Macrophages handle the engulfed mycobacteria by a number of mechanisms that include the generation of reactive oxygen and nitrogen intermediates and by the growth inhibition/killing action of cytokines such as IFN- $\gamma$ and TNF- $\alpha$, although many of the former mechanisms are mostly effective only in animal models and their role in humans requires further elaboration. ${ }^{23}$ Importantly, the mechanism behind so-called "caseous necrosis" which is basic to the tuberculous disease remains largely unknown. ${ }^{4}$

More recently there has been great interest in the apoptosis of macrophages as a method of in vivo defence against mycobacterial infection..$^{5-8}$ Apoptosis is thought to be an important defence mechanism for the destruction of infected macrophages. While apoptosis of HIV-infected and bystander CD4+ and CD8+ is well studied, ${ }^{10}$ as is the role of macrophages in inducing apoptosis of $\mathrm{T}$ lymphocytes, ${ }^{11}{ }^{12}$ there are few reports on apoptosis in infected macrophages, ${ }^{13-15}$ and none relate to caseating granulomas.

This study was designed to examine if apoptosis has a significant role in the production of so-called caseous necrosis in lymph nodes excised from patients with clinically confirmed HIV infection, the co-infection of tuberculosis and HIV being well recognised. ${ }^{16}{ }^{17}$ In addition, the lymph node sections were immunostained for some apoptosisrelated proteins in order to understand their relationship to the distribution of $B$ and $T$ lymphocytes, and macrophages in the granulomatous response to mycobacterial infection. 
Table 1 Immunostaining of lymphocytes and macrophages constituting the granulomatous response in mycobacterial lymphadenopathy

\begin{tabular}{|c|c|c|c|c|c|c|c|c|c|c|}
\hline & Casp & TUNEL & CD20 & CD3 & CD8 & CD4 & Bax & Fas & Bcl2 & AFB \\
\hline Caseating granulomas $(n=30)$ & +++ & +++ & 0 & +++ & +++ & 0 & +++ & +++ & 0 & Occ \\
\hline Extensive necrosis $(n=5)$ & ++ & + & 0 & ++ & ++ & 0 & +++ & +++ & 0 & $0 c c$ \\
\hline Sarcoid-like $(n=5)$ & + & + & + & ++ & ++ & ++ & +++ & +++ & 0 & Mod \\
\hline Foamy macrophages $(n=5)$ & + & + & 0 & ++ & ++ & 0 & +++ & +++ & 0 & Abund \\
\hline Inflammatory pseudotumour $(\mathrm{n}=3)$ & + & + & + & + & + & 0 & +++ & +++ & 0 & Abund \\
\hline Lymphoid hyperplasia $(n=7)$ & NA & NA & +++ & +++ & +++ & +++ & $*$ & * & 0 & None \\
\hline
\end{tabular}

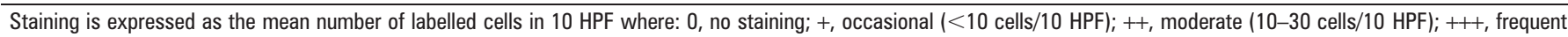
(>30 cells/10 HPF).

*Positive staining was observed in scattered epithelioid cells in the cases with non-specific lymphoid hyperplasia.

AFB, acid fast bacillus; Occ, occasional; Mod, moderate numbers; Abund, abundant; NA, not appropriate; Casp, caspase 3;

\section{MATERIALS AND METHODS}

Lymph node biopsy specimens from a previously published series of 55 HIV-infected Thai patients obtained through a search of the files of the Department of Pathology at Chulalongkorn Hospital, Bangkok and Hi-Tech Laboratory, Bangkok, Thailand, between 1 January 1997 and 30 August 1998 were studied. All biopsy specimens were from the head and neck region; HIV infection was confirmed clinically and by antibody detection through ELISA with confirmation by immunofluorescent assay (IFA) or western blot. In a previous study all cases had been examined with H\&E, Ziehl-Neelsen, Gram, periodic acid-Schiff (PAS), PAS with diastase, and Grocott methenamine silver stains; the morphological changes seen were divided into six inflammatory patterns: 1, classic caseating granulomas (30 cases); 2, extensive necrosis with minimal residual granulomatous response (5 cases); 3, sarcoidlike non-caseating granulomas (5 cases); 4 , foamy macrophage response (5 cases); 5 , inflammatory pseudotumour-like response (3 cases); and 6, non-specific lymphoid hyperplasia (7 cases). ${ }^{18}$ The patients were not receiving anti-mycobacterial therapy at the time of biopsy. All were clinically cleared of pulmonary tuberculosis and chest $x$-rays were clear.

In this study, immunostains were performed following microwave antigen retrieval at $98^{\circ} \mathrm{C}$ for 20 minutes, ${ }^{19}$ antibody, source, dilution, and retrieval solution as follows: caspase 3 (Cell Signalling, Beverly, Massachusetts, USA; 1:200, citrate/EDTA, pH 8.0), CD20 (Dako, Sydney, Australia; 1:400, citrate buffer at pH 6.0), CD3 (Novocastra, Mt Waverley, Victoria, Australia; 1:15, citrate buffer at $\mathrm{pH} 6.0$ ), CD4 (Novocastra; 1:75, citrateEDTA buffer at $\mathrm{pH} 8.0$ ), CD8 (Novocastra; 1:300, citrate-EDTA buffer at pH 8.0), CD68 (Dako; 1:50, citrate buffer at pH 6.0), Bcl-2 (Dako; 1:50, citrate buffer at pH 6.0), Bax (Serotec, Sydney, Australia; 1:100, citrate buffer pH 6.0), Fas (CD95) (Santa Cruz Biotechnology, Santa Cruz, California, USA; 1:50, citrate/EDTA pH 8.0). A standard streptavidin biotin peroxidase technique was employed ${ }^{20}$ with diaminobenzidine as the chromogen and Mayer's haematoxylin as counterstain. TUNEL (terminal deoxynucleotidyl transferase-mediated deoxyuridine triphosphate nick end-labelling) was performed with the Boeheringer Mannheim in situ cell death detection kit (Cat. No. 1684817, Mannheim, Germany) adhering strictly to the manufacturer's instructions. Positive and negative controls were employed for all stains. The extent of staining was scored in a semi-quantitative manner as the mean number of positively labelled cells after counting 10 high power fields (HPFs) in the most strongly staining areas, where 0 , no staining; +, occasionally ( $<10$ cells per $\mathrm{HPF})$; ++ , moderate $(10-30$ cells per $\mathrm{HPF}),+++$, frequent $(>30$ cells per HPF). Similar counts were made for apoptotic bodies in $\mathrm{H} \& \mathrm{E}$ stained sections. Material from non-HIV-infected patients with classic caseating granulomas in cervical nodes $(n=3)$ and lung $(n=2)$ were also stained for caspase 3 as controls.

\section{RESULTS}

The spectrum of histological changes in this series of cases has previously been described. ${ }^{18}$ In the context of the present study, it should be noted that the category of extensive necrosis with minimal granulomatous reaction was not homogenous and often included an occasional sarcoid-like granulomas, sometimes with small areas of necrosis. Two of the five nodes that showed extensive necrosis also displayed extensive fibrosis. Table 1 shows results of semi-quantitative assessment of the immunostains.

\section{Apoptosis}

Apoptosis could be demonstrated in all lymph nodes showing a granulomatous response. Extensive apoptosis was seen in classic caseating necrosis where apoptotic bodies filled the entire caseous centre of the granulomas (fig 1). This was confirmed by both caspase 3 immunostaining (fig 2A) and TUNEL. In extensive confluent caseation varying numbers of apoptotic bodies were located at the periphery of the necrotic areas (fig 2B). Cases with sarcoid-like granulomas invariably revealed foci of apoptosis of variable size readily highlighted by caspase 3 immunostaining (fig 2C). Focal collections of apoptotic bodies could be identified in cases with psudoinflammatory tumourlike response; areas of variable size with apoptosis were seen in

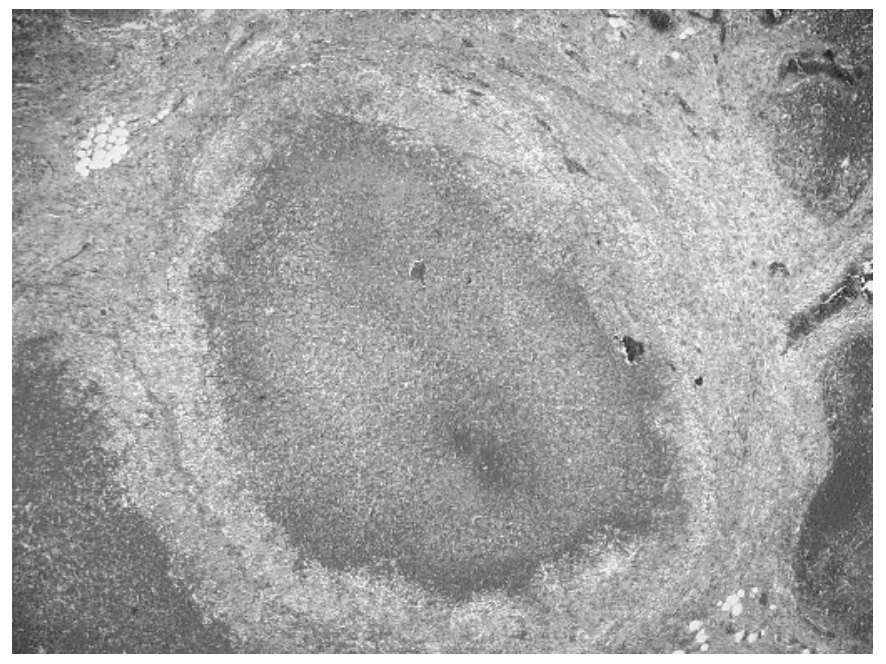

Figure 1 A large caseating granuloma with a centre composed of apoptotic bodies is surrounded by a paler layer of epithelioid cells and an outermost rim of fibrous tissue. Another similar granuloma occupies the lower left of the field. 
Figure 2 Caspase 3 immunostaining highlighting apoptotic cells $(A)$ filling the centre of a caseating granuloma, $(B)$ in the periphery of an "old" granulomas with extensive caseation and fibrosis, (C) in sarcoid-like granulomas, and (D) focally in the foamy macrophage reaction.
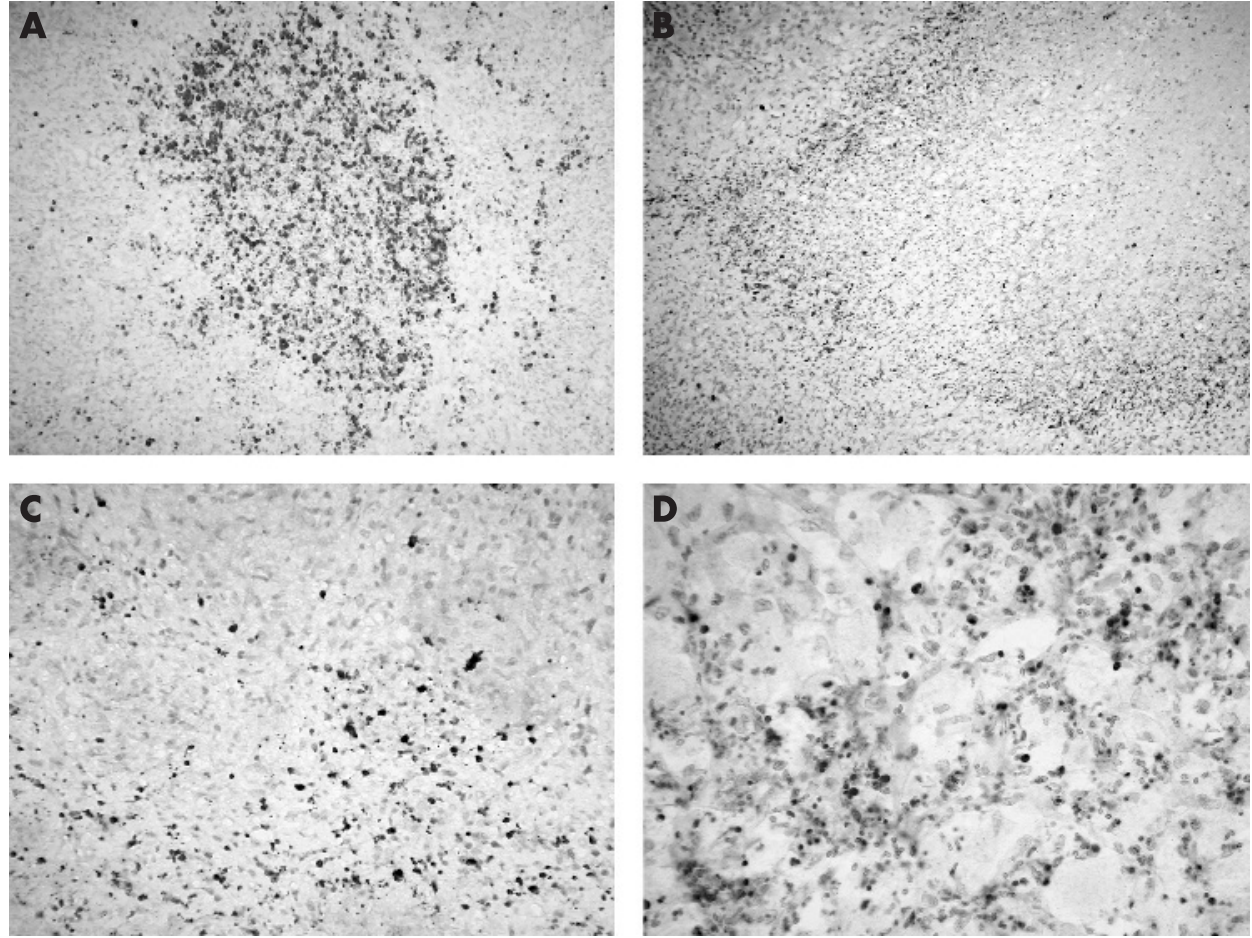

those nodes with a foam cell response (fig 2D) and in those with diffuse granulomatous response. Only the cases of non-specific lymphoid hyperplasia did not display apoptosis (other than in germinal centres), although in those sections where there were areas with epithelioid cells, occasional caspase 3 positive cells could be identified. TUNEL staining was more variable and there was often background staining within the necrotic areas and morphologically distinct apoptotic bodies occasionally failed to stain. Nonetheless, the staining with TUNEL paralleled that obtained with caspase 3 , but to a lesser extent.

The controls of non-HIV-infected patients showed similar staining for caspase 3 in caseating granulomas from both lymph nodes and lung (fig 3).

\section{T and B lymphocytes, and macrophages}

Staining for CD3 and CD8 revealed that the majority of lymphocytes associated with the apoptosis were positive for these antigens. CD8+ cells lined caseating centres and were present within the areas of caseation (fig 4A); they were intimately related to the smaller foci of apoptosis (fig 4B). The nodes were largely depleted of CD4+ cells; CD20+ cells were confined to areas of residual small lymphocytes and germinal centres separate from the granulomatous response and caseation. Interestingly, besides CD68, free CD3 and CD8 antigen was present in the areas of caseation and apoptotic bodies with intact cell membranes stained for these antigens. Double staining for caspase 3 and CD4, and caspase 3 and CD8 provided inconclusive results as the disruption of cell membranes and the release of lymphocyte antigens made interpretation difficult (data not provided). CD8+ $\mathrm{T}$ cells were most frequent in the immediate vicinity of granulomas in those cases with classic caseating granulomas. Moderate numbers of such cells were associated with nodes displaying extensive necrosis, sarcoid-like granulomas and foamy macrophages, whereas those with inflammatory pseudotumour response had only occasional scattered lymphocytes (table 1).

\section{Bax, Fas, and Bcl-2}

All granulomas failed to show Bcl-2 immunoexpression, although labelling was evident in small B-lymphocytes in the periphery of the granulomatous response. Almost all epithelioid cells forming granulomas and surrounding areas of necrosis displayed diffuse and strong labelling for both Bax (fig 5A,B) and Fas (fig 6). Scattered epithelioid cells in cases of non-specific lymphoid hyperplasia also showed staining for these two antigens, as did foamy macrophages and the spindle cells of the inflammatory pseudo-tumour pattern of response. All epithelioid cells were negative for Bcl-2.

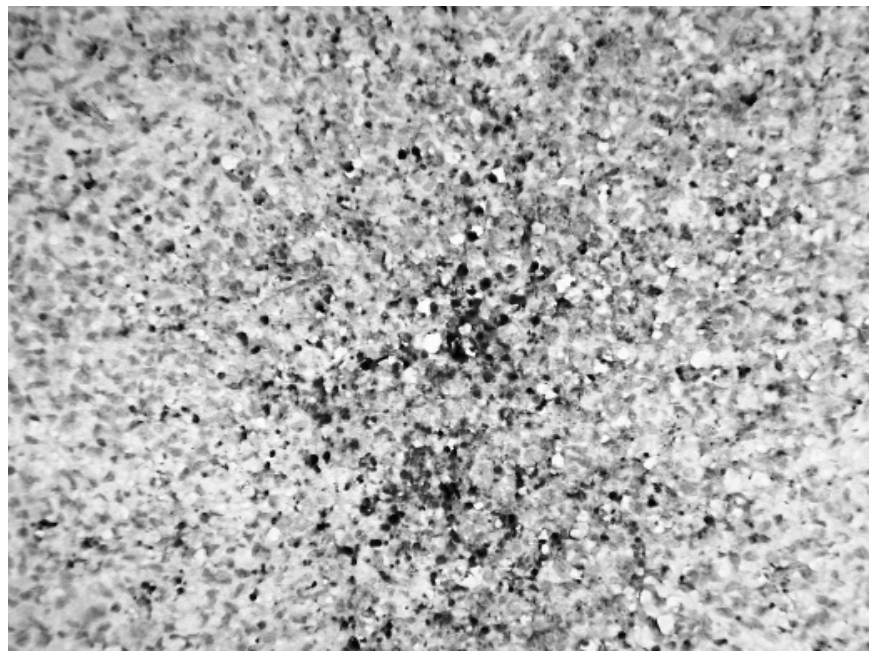

Figure 3 Caspase 3 immunostaining showing extensive apoptosis in a classical caseating granuloma in the lung of a non-HIV-infected patient. The extent of staining was very similar to that seen in HIV-infected cases. 
Figure $4 \quad \mathrm{CD} 8+\mathrm{T}$ cells are shown $(\mathrm{A})$ infiltrating among epithelioid macrophages as well as the caseous centre (indicated by " $\mathrm{C}$ ") of a caseating granuloma, and (B) closely associated with small foci of apoptosis in a node with foamy macrophages.
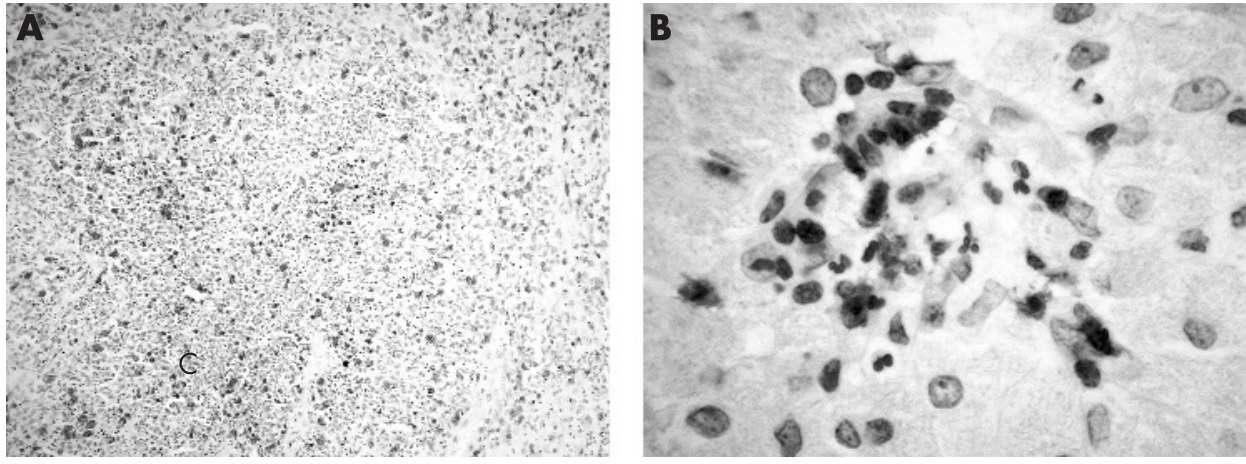

\section{DISCUSSION}

The classic description of caseous necrosis from a standard pathology textbook states that it is "a distinctive form of coagulative necrosis, encountered most often in foci of tuberculous infections...the necrotic focus appears as amorphous granular debris seemingly composed of fragmented, coagulated cells and amorphous granular debris enclosed within a distinctive inflammatory border known as a granulomatous reaction. Unlike coagulative necrosis, the tissue architecture is completely obliterated". "This study confirms the previous suggestion by Fayyazi et al ${ }^{21}$ that granulomatous caseation is associated with apoptosis. Our demonstration that apoptosis as identified by its distinctive morphological features and by immunostaining for caspase 3 and TUNEL can be so extensive as to fill entire areas of caseation indicates for the first time that it may be entirely responsible for the so-called "necrosis" seen in mycobacterial granulomas. Variable numbers of apoptotic bodies and caspase 3 labelled nuclei were also present in granulomas with extensive and confluent necrosis. Unlike Fayyazi et $a^{21}$ who did not find apoptosis in this type of granulomas with the TUNEL technique, we were able to show residual apoptotic nuclei with caspase 3 . In lymph nodes with a foamy macrophage response akin to the lepra cells in lepromatous leprosy and those with a pseudo-inflammatory tumour response, apoptosis was also present but focal.

Apoptosis, or programmed cell death, is an energy dependent process that involves the activation of caspases (including caspase 3). Cell shrinkage and fragmentation occurs swiftly and adjacent cells and macrophages rapidly phagocytose the apoptotic debris so that considerable apoptosis needs to occur before it can be recognised morphologically; furthermore, evidence of the process is also rapidly removed. Because apoptotic bodies are transient it is not surprising that regressive granulomas may no longer display features of apoptosis. Indeed the ability to identify apoptosis is determined by time and "older" lesions may no longer show evidence of the process. Clearly, apoptosis also occurs in all the other reaction patterns associated with mycobacterial lymphadenitis as shown in our cases but in smaller foci. Apoptosis has also been reported in the granulomas of cat scratch disease $e^{22}$ but has not been previously demonstrated to the extent seen in our cases. It has been the conventional teaching for decades that caseation is the result of necrosis. However, the absence of a neutrophilic inflammatory response and the very localised nature of caseation together with our demonstration of apoptosis, which can comprise the entire area of caseation, would militate against this age-old contention. So-called "caseous necrosis" can be entirely the result of apoptosis.

There is an increasing realisation that apoptosis of host macrophages has a significant role in the response to mycobacterial infection, ${ }^{23}{ }^{24}$ although the beneficial effects of the socalled "necrosis" of tubercles is the subject of some debate. The necrotic debris is said to harbour free viable bacilli that escape the antimicrobial activity of phagocytes and are protected against chemotherapy. Furthermore, it has been observed that the lesions are themselves irreversible and cause considerable damage to the lung and other infected tissue, including cavitation and erosion of vessels and bronchi leading to haematogenous and aerosol spread of the bacilli respectively. On the other hand, it has been argued that granuloma formation is necessary to contain the bacilli and may be responsible for the long dormancy associated with $M$ tuberculosis. ${ }^{3}$ Importantly, it is now realised that the organisms have developed the capacity to escape killing mechanisms in human macrophages, which serve as a privileged site for mycobacterial proliferation.

This is the first study of human mycobacterial lymphadenitis, which employs immunostaining for caspase 3 to confirm that the "karyorrhexic" debris observed in caseating granulomas is the result of apoptosis. It confirms a previous report where
Figure 5 Immunostaining for Bax showing strong immunoexpression in (A) epithelioid macrophages in a caseating granuloma (caseating area indicated by “ $\mathrm{C}$ ") and (B) in foamy macrophages.
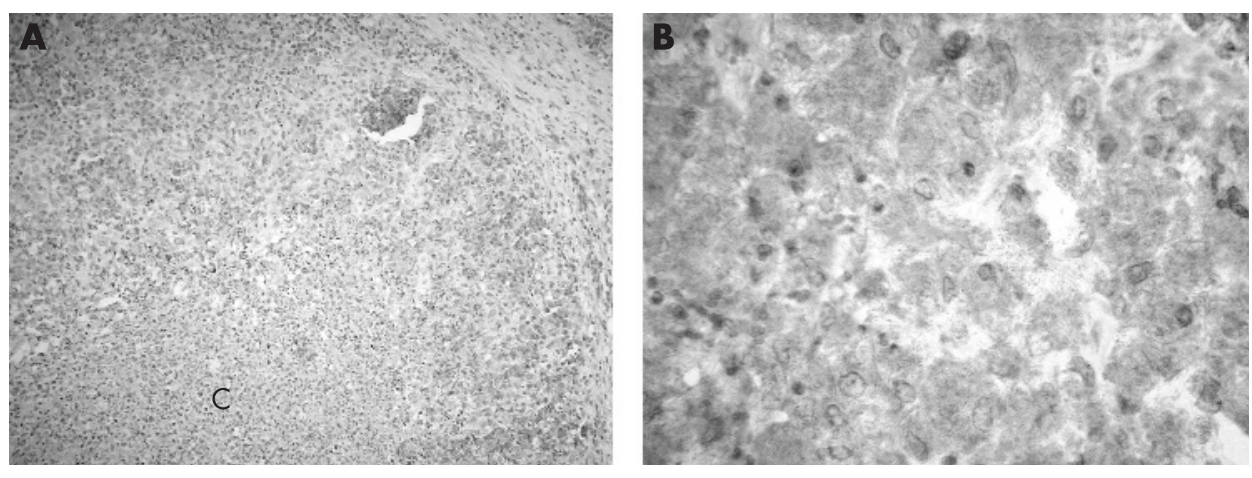


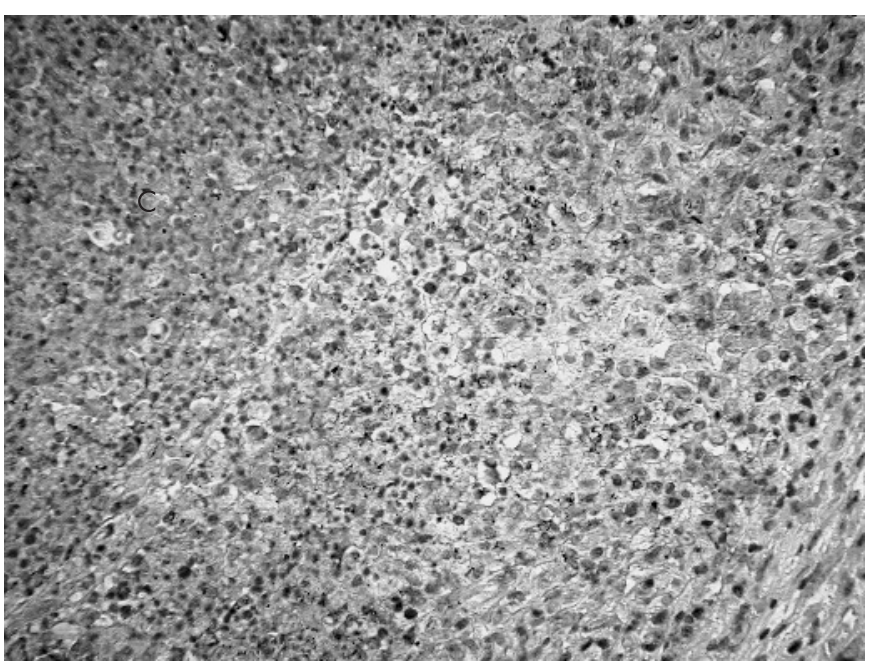

Figure 6 Diffuse immunoexpression of Fas is present in the epithelioid macrophages of a caseating granuloma; " $C$ " identifies the caseous centre.

apoptosis was detected by TUNEL. ${ }^{21}$ The accuracy of TUNEL has been called into question as the method of fixation and pretreatment can affect the detection of DNA strand breaks, and importantly, TUNEL cannot always distinguish apoptotic and necrotic cells and may falsely label cells in the process of DNA repair. ${ }^{25}$ Caspase 3 immunostaining is the preferred method that allows for rapid and consistent quantification, especially at the peak of apoptosis when TUNEL detected only low levels of fragmented DNA. ${ }^{25}$ The variation in the extent of apoptosis among the reaction patterns seen in our cases reflected the bacterial load in the macrophages and thus the host's ability to contain the organism. Cases with foamy macrophages and spindle cell response contained abundant acidfast organisms and showed the least apoptotic activity compared to those with caseating granulomas and confluent necrosis where organisms were generally difficult to find and when present were extracellular. In sarcoid-like reactions with small foci of apoptosis, mycobacteria were readily detected in clusters similar to that described in $M$ leprae. ${ }^{5}$ The apoptosis demonstrated by caspase 3 staining in caseating granulomas from non-HIV-infected patients in both lymph nodes and lung was similar if not identical in extent to that seen in similar lesions in the HIV-infected patients. These cases were clinically considered to be due to $M$ tuberculosis and strongly support the contention that the so-called necrosis in mycobacterial granulomas may largely be due to apoptosis.

The other question that we sought to answer in this study was the relationship of apoptosis-related antigens to the $B$ and $\mathrm{T}$ cells and macrophages in the granulomas. We confirmed previous observations that the majority of CD68+ macrophages surrounding caseous areas were negative for the anti-apoptotic protein Bcl-2 but strongly positive for the pro-apoptotic proteins Bax and Fas. ${ }^{21}$ We also showed that CD20+ B cells do not participate in the granuloma formation and that the lymphocytes associated with granulomas are CD3+ CD8+ T cells. Both these antigens as well as CD68 were present in and among the apoptotic bodies, suggesting that CD8+ T cells as well as CD68+ macrophages undergo apoptosis. These observations are not without precedent. It has been thought that mycobacteria, like other intracellular pathogens, induce antigenspecific, MHC-restricted CD4+ and CD8+ T cells, with the bulk of $M$ tuberculosis-specific activity in humans mediated by CD4+ cells which effect the killing of infected macrophages, thus destroying the intracellular habitat of the microorganism. The introduction of soluble recombinant Fas-ligand (FasL) has been shown to induce apoptosis of mycobacterium-infected human macrophages in vitro, producing a substantial reduction of bacillary viability. Apoptosis of infected macrophages was also induced by TNF- $\alpha{ }^{26}$ The role of CD4+ T cells in this process was also supported by a mouse model where the appearance of "necrosis" could be blocked by the administration of antibodies specific to CD4 or IFN- $\gamma \cdot{ }^{18}$

Fas, FasL, Bax and Bcl-2 are apoptosis-regulating proteins that play an important role in apoptosis and the immune response. ${ }^{26}$ In murine models, it has been demonstrated that the expression of these proteins are exploited by mycobacteria to evade the host's immune response. By virtue of increased Bcl-2 and reduced Bax expression, infected cells evade activation-induced apoptotic cell death. In addition, it has been suggested that the infected macrophages can induce apoptosis of surrounding lymphocytes by increased expression of FasL and reduced expression of Fas. $^{28} 29$ These findings in murine tissues are contrary to those obtained in our study where the pro-apoptosis proteins Bax and Fas were strongly expressed and Bcl-2 was not stained in all epithelioid granulomas irrespective of the pattern of histological response. Similar observations in tuberculosis have been reported in human tissues. ${ }^{21}$ It would thus appear that the proposed mechanism for immune evasion in murine tuberculosis is not applicable to humans and that some other mechanism of immune evasion is adopted by the mycobacteria in the human.

Unlike the cases reported by Fayyadi et al, ${ }^{21}$ our biopsy specimens were from HIV-infected patients and all displayed a reduction or absence of CD4+ cells in the nodes. While a cellmediated immune response is crucial for control of intracellular infection, the extensive apoptosis in our cases indicates that the CD4+ T cell-mediated IFN- $\gamma$ mechanism of apoptosis previously proposed $^{18}$ may not be the sole method to activate apoptosis and an alternative mechanism exists. Recently, it has been suggested that CD8+ $\mathrm{T}$ cells may have a protective role against tuberculosis. $^{3031}$ Besides the secretion of perforin and granzymes, CD8+ T cells are also capable of secreting cytokines such as IFN- $\gamma$ and TNF- $\alpha$, the former activating macrophages as well as killing mycobacteria. The role of CD8 $+\mathrm{T}$ cells in protecting against tuberculosis has been established by experimental infection of mice lacking CD8+ $\mathrm{T}$ cells. ${ }^{32}{ }^{33}$ Cross-priming of CD8+ T cells has been demonstrated by the release of apoptotic vesicles from infected macrophages that are carried by uninfected dendritic cells which, in turn, present the antigens through $\mathrm{MHC}-1$ and $\mathrm{CD} 1 \mathrm{~b}$ to $\mathrm{CD} 8+{ }^{34}$ and $\mathrm{CD} 4+\mathrm{T}$ cells. ${ }^{31}$ Activated CD8+ cells can thus, through perforin and granzymes and the expression of FasL activate apoptosis in the infected macrophages. In this regard, it is interesting to note that CD8+ $\mathrm{T}$ cells were intimately related to the apoptotic bodies in our cases.

FasL-induced apoptosis of human macrophages has been shown to reduce the viability of infecting $M$ tuberculosis, ${ }^{26}{ }^{35}$ and the induction of apoptosis of mycobacterium-infected cells by other agents such as $\mathrm{H}_{2} \mathrm{O}_{2}{ }^{36}$ and adenosine triphosphate ${ }^{37}$ lead to the elimination of the infecting organism. Importantly, it was shown that the inhibition and prevention of spread of $M$ avium-intracellulare (MAC) is due to apoptosis and not necrosis of infected macrophages. ${ }^{37}$ It is interesting that infection with $M$ bovis $\mathrm{BCG}^{38}$ and $M$ leprae $^{39}$ has also been shown to result in 


\section{Take-home messages}

- So-called caseous necrosis in classical mycobacterial granulomas is largely due to apoptosis.

- The extent of apoptosis varies inversely with the mycobacterial load.

- Apoptosis of macrophages harbouring mycobacteria may be a method of exposing bacterial antigen which is otherwise "hidden" from the immune system.

- Apoptosis of macrophages occurs in the absence of CD4+ cells, suggesting an alternative pathway for inducing this form of cell death, perhaps via CD8+ lymphocytes.

- Caspase 3 immunostaining is a more representative reflection of apoptosis than TUNEL.

increased macrophage apoptosis, and apoptosis limits the growth of $\mathrm{BCG}^{40}$ and $M$ tuberculosis in human monocytes. ${ }^{41}$

With the advent of highly active antiretroviral therapy (HAART), the immune restoration inflammatory syndrome (IRIS) may develop. This currently poorly defined syndrome has as an essential criterion, a change or deterioration of the patient's health status as a result of a new or pre-existing pathogen that commonly includes tuberculosis, or infection with MAC, Cryptococcus neoformans or viruses such as herpes and hepatitis viruses. ${ }^{42}$ IRIS was not diagnosed in any of the patients in this series. While the species of infecting acid-fast bacillus was not identified in our cases, MAC was considered the most likely organism given the clinical and morphological features, particularly in those cases with the extensive foam cell and the inflammatory pseudo-tumour response, both patterns having not been described in association with other species of mycobacteria. Even if other species were responsible for some of the cases in our series, it does not detract from the observation that apoptosis is a significant if not the primary cause of so-called "caseous necrosis". Clearly in a study based on fixed paraffin-embedded human tissues such as ours, it is not possible to fully elucidate all the answers concerning the mechanism of apoptosis in the setting of mycobacterium infection. It appears valid to conclude that the strong expression of Bax and Fas, and the absence of staining for $\mathrm{Bcl}-2$ negates the mechanism of FasL-induced apoptosis demonstrated in the murine model. In our CD4+ T cell-deficient cases, IFN- $\gamma$ was unlike to be a significant mediator of apoptosis.

Our results do not allow us to speculate on the nature of the apoptosis observed, but it is relevant to note that the extent of programmed cell death appears to be inversely related to bacteria load; it would be appropriate to suggest that the stimulation of apoptosis in infected macrophages could be therapeutically exploited in mycobacterial infections. The elucidation of the precise mechanism of activating apoptosis of infected macrophages in CD4-deficient patients may also provide answers for therapeutic manipulations in the treatment of mycobacterial infections, especially in HIV-infected and CD4 deficient individuals.

Competing interests: None.

\section{REFERENCES}

1. Kumar V, Abbas AK, Fausto N. Robbins and Cotran_-Pathologic basis of disease, 7th edn. Philadelphia, PA: Elsevier Saunders, 2005:22.

2. Raja A. Immunology of tuberculosis. Indian J Med Res 2004;120:213-32.

3. Saunders BM, Britton WJ. Life and death in the granuloma: immunopathology of tuberculosis. Immunol Cell Biol 2007;85:103-11.
4. Mustafa T, Mogga SJ, Mfinnangaa SGM, et al. Immunohistochemical analysis of cytokines and apoptosis in tuberculous lymphadenitis. Immunology

2005:117:454-62.

5. Harries AD, Dye C. Tuberculosis. Ann Trop Med Parasitol 2006;100:415-31.

6. Ahmed AB, Abubakar I, Delpech V, et al. The growing impact of HIV infection on the epidemiology of tuberculosis in England and Wales. Thorax Online thx.2006.072611v1

7. Holm GH, Gabuzda D. Distinct mechanisms of CD4+ and CD8+ T-cell activation and bystander apoptosis induced by human immunodeficiency virus type 1 virions. J Virol 2005; 79:6299-311.

8. Espert L, Denizot M, Grimaldi M, et al. Autophagy is involved in T cell death after binding of HIV-1 envelope proteins to CXXR4. J Clin Invest 2006;116:2161-72.

9. Ross TM. Using death to one's advantage: HIV modulation of apoptosis. Leukemia 2006; 15:332-41.

10. Mahlknecht U, Deng C, Lu MC, et al. Resistance to apoptosis in HIV-infected CD4+ T lymphocytes is mediated by macrophages: role for Nef and immune activation in viral persistence. J Immunol 2000;165:6437-46.

11. Cosenza MA, Zhao M-L, Lee SC. HIV-1 expression protects macrophages and microglia from apoptotic death. Neuropath Appl Neurol 2004;30:478-90.

12. Huang $\mathbf{Y}$, Erdmann $\mathrm{N}$, Peng $\mathrm{H}$, et al. TRAlL-mediated apoptosis in HIV1-infected macrophages is dependent on the inhibition of Akt-1 phosphorylation. J Immunol 2006:177:2304-13

13. Kim N, Dabrowska A, Jenner RG, et al. Human and simian immunodeficiency virusmediated upregulation of the aopototic factor TRAIL occurs in antigen-presenting cells from AIDS-susceptible but not from AIDS-resistant species. J Virol 2007;81:7584-97.

14. Walsh DS, Lane JE, Abalos RM, et al. TUNEL and limited immunophenotypic analyses of apoptosis in paucibacillary and multibacillary leprosy lesions. FEMS Immunol Med Microbiol 2004;41:265-9.

15. Zhang J, Jiang R, Takayama H, et al. Survival of virulent Mycobacterium tuberculosis involves preventing apoptosis induced by Bcl-2 upregulation and release resulting from necrosis in $\mathrm{J} 774$ macrophages. Microbiol Immunol 2005;49:845-52.

16. Bocchino M, Galati D, Sanduzzi A, et al. Role of mycobacteria-induced monocyte/ macrophage apoptosis in the pathogenesis of human tuberculosis. Int J Tuberc Lung Dis 2005:9:375-83.

17. Rios-Barrera VA, Campos-Pena V, Aguilar-Leon D, et al. Macrophage and T lymphocyte apoptosis during experimental pulmonary tuberculosis: their relationship to mycobacterial viorulence. Eur J Immunol 2006;36:345-53.

18. Wannakrairot $\mathbf{P}$, Leong TY-M, Leong AS-Y. The morphological spectrum of lymphadenopathy in HIV infected patients. Pathology 2007;39:223-7

19. Leong AS-Y, Milios J. An assessment of the efficacy of the microwave-antigen retrieval procedure on a range of tissue antigens. Applied Immunohistochem 1993;1:267-74.

20. Leong AS-Y, Milios J. Staining protocols; antibodies, sources, dilutions and costs. In Leong AS-Y, ed. Applied immunohistochemistry for the surgical pathologist. London: Arnold, 1993:385-401.

21. Fayyazi A, Eichmeyer B, Schweyer S, et al. Apoptosis of macrophages and T cells in tuberculosis associated caseous necrosis. J Pathol 2000;191:417-25.

22. Schweyer S, Fayyazi A. Activation and apoptosis of macrophages in cat scratch disease. J Pathol 2002;198:534-40.

23. Keane J, Remold HG, Kornfeld H. Virulent Mycobacterium tuberculosis strains evade apoptosis of infected alveolar macrophages. J Immunol 2000;164:2016-21.

24. Sly LM, Higley-Wilson SM, Reiner NE, et al. Survival of Mycobacterium tuberculosis in host macrophages involves resistance to apoptosis dependent upon induction of antiapoptotic Bcl-2 family member Mcl-1. J Immunol 2003;170:430-7.

25. Watanabe $\mathbf{M}$, Hitomi $\mathbf{M}$, van der Wee $\mathrm{K}$, et al. The pros and cons of apoptosis assays for use in the study of cells, tissues and organs. Microsc Microanal 2002;8:375-91

26. Oddo M, Renno T, Attinger $\mathrm{A}$, et al. Fas ligand-induced apoptosis of infected human macrophages reduces the viability of intracellular Mycobacterium tuberculosis. J Immunol 1998; 160:5448-54.

27. Florido M, Cooper AM, Appelberg R. Immunological basis of the development of necrotic lesions following Mycobacterium avium infection. Immunology 2002:106:590-601.

28. Mogga SJ, Mustafa T, Sviland L, et al. Increased Bcl-2 and reduced Bax expression in infected macrophages in slowly progressive primary murine Mycobacterium tuberculosis infection. Scand J Immunol 2002;56:383-91.

29. Mustafa T, Bjune TG, Jonsson R, et al. Increased expression of fas ligand in human tuberculosis and leprosy lesions: a potential novel mechanism of immune evasion in mycobacterial infection. Scand J Immunol 2001;54:630-9.

30. Brookes RH, Pathan AA, McShane $\mathrm{H}$, et al. $\mathrm{CD}^{+} \mathrm{T}$ cell mediated suppression of intracellular Mycobacterium tuberculosis growth in activated human macrophages. Eur J Immunol 2003;33:3293-302.

31. Winau F, Weber S, Sad S, et al. Apoptotic vesicles crossprime CD8 T cells and protect against tuberculosis. Immunity 2006;24:105-17.

32. Behar SM, Dascher CC, Grusby MJ, et al. Susceptibility of mice deficient in CD1d or TAP1 to infection with Mycobacterium tuberculosis. J Exp Med 1999;189:1973-80.

33. Rolph MS, Raupach B, Kobernick HH, et al. MHC class la-restricted T cell primarily account for beta 2-microglobulin-dependent resistance to Mycobacterium tuberculosis. Eur J Immunol 2001;31:1944-9.

34. Schaible UE, Wnnau F, Sieling PA, et al. Apoptosis facilitates antigen presentation to T lymphocytes through MHC-1 and CD1 in tuberculosis. Nature Med 2003;9: 1039-46. 
35. Bermudez LEM, Young LS. Tumor necrosis factor, alone or in combination with IL-2, but not IFN- $\gamma$ is associated with macrophage killing of Mycobacterium avium complex. J Immunol 1988;140:3006-13.

36. Laochumroonvorapong $\mathbf{P}$, Paul $\mathrm{S}$, Elkin $\mathrm{KB}$, et al. $\mathrm{H}_{2} \mathrm{O}_{2}$ induces monocyte apoptosis and reduces viability of Mycobacterium avium-M. intracellulare within cultured human monocytes. Infect Immunol 1996;64:452-9.

37. Lammas DA, Stober C, Harvey CJ, et al. ATP-induced killing of mycobacteria by human macrophages is mediated by purinergic P2Z (P2X7) receptors. Immunity 1997; 7:433-44

38. Klingler $\mathbf{K}$, Tchou-Wong KM, Brandli 0 , et al. Effects of mycobacteria on regulation of apoptosis in mononuclear phagocytes. Infect Immunol 1997;65:5272-8.
39. Hernandez M0, Neves I Jr, Sales JS, et al. Induction of apoptosis in monocytes by Mycobacerium leprae in vitro: a possible role for tumour necrosis factor- $\alpha$. Immunology 2003:109:156-64.

40. Molloy A, Laochumroonvorapong P, Kaplan G. Apoptosis, but not necrosis, of infected monocytes is coupled with killing of intracellular Bacillus Calmette-Guerin. J Exp Med 1994;180:1499-509.

41. Oddo M, Renno T, Attinger A, et al. Fas ligand-induced apoptosis of infected human macrophages reduces the viability of intracellular Mycobacterium tuberculosis. $\mathrm{J}$ Immunol 1998:160:5448-54.

42. Lipman M, Breen R. Immune reconstitution inflammatory syndrome in HIV. Curr Opin Infect Dis 2006;19:20-5.

\section{Take advantage of BMJ Journals' remarkable catalogue of titles with Related Collections}

No busy professional has time to browse through all pertinent journals to find relevant articles, but with Related Collections you no longer have to. Follow the "Related Collections" link from any article and use the "Show Collections from other Journals" to expand your search across all BMJ Journals. Or simply follow the "Browse by topic" link on the home page. By setting up your own collections and receiving email alerts every time an article is added to your chosen area, you can build up your own significant body of knowledge. 